\title{
Serological Evidence of Lyssavirus Infection among Bats in Nagaland, a North-Eastern State in India
}

\author{
R. S. MANI ${ }^{1}$, D. P. DOVIH ${ }^{2}$, M. A. ASHWINI ${ }^{1}$, B. CHATTOPADHYAY ${ }^{2} \dagger$, \\ P. K. HARSHA ${ }^{1}$, K. M. GARG ${ }^{2} \dagger$, S. SUDARSHAN ${ }^{1}$, R. PUTTASWAMAIAH ${ }^{3}$, \\ U. RAMAKRISHNAN ${ }^{2}$ AND S. N. MADHUSUDANA ${ }^{1}+$ \\ ${ }^{1}$ Department of Neurovirology, WHO Collaborating Centre for Reference and Research on Rabies, \\ National Institute of Mental Health \& Neurosciences (NIMHANS), Bangalore, Karnataka, India \\ ${ }^{2}$ Ecology and Evolution, National Centre for Biological Sciences, Tata Institute of Fundamental Research, \\ Bangalore, Karnataka, India \\ ${ }^{3}$ Bat Conservation India Trust, Bangalore, Karnataka, India
}

Received 10 September 2016; Final revision 18 January 2017; Accepted 24 January 2017; first published online 23 February 2017

\section{SUMMARY}

Bats are known to be reservoirs of several medically important viruses including lyssaviruses. However, no systematic surveillance for bat rabies has been carried out in India, a canine rabies endemic country with a high burden of human rabies. Surveillance for rabies virus (RABV) infection in bats was therefore carried out in Nagaland, a north-eastern state in India at sites with intense human-bat interfaces during traditional bat harvests. Brain tissues and sera from bats were tested for evidence of infection due to RABV. Brain tissues were subjected to the fluorescent antibody test for detection of viral antigen and real-time reverse transcriptase PCR for presence of viral RNA. Bat sera were tested for the presence of rabies neutralizing antibodies by the rapid fluorescent focus inhibition test. None of the bat brains tested $(n=164)$ were positive for viral antigen or viral RNA. However, rabies neutralizing antibodies were detected in 4/78 (5.1\%) bat sera tested, suggesting prior exposure to RABV or related lyssaviruses. The serological evidence of lyssaviral infection in Indian bats may have important implications in disease transmission and rabies control measures, and warrant extensive bat surveillance to better define the prevalence of lyssaviral infection in bats.

Key words: Rabies (animal), rabies (human), zoonoses.

\section{INTRODUCTION}

Rabies is a fatal encephalomyelitis caused by the rabies virus (RABV) and some other viruses in the Lyssavirus genus of family Rhabdoviridae.

\footnotetext{
* Author for correspondence: Dr. R. S. Mani, Department of Neurovirology, WHO Collaborating Centre for Reference and Research on Rabies, National Institute of Mental Health \& Neurosciences (NIMHANS), Bangalore-560029, Karnataka, India. (Email: drreeta@gmail.com)

$\dagger$ Current Address: National University of Singapore, Singapore.

\$ Deceased
}

Bats are recognized as important reservoirs for many viruses, including lyssaviruses. In the Americas, only variants of the classical RABV, which is responsible for a majority of human rabies cases across the globe, have been detected in both bats and terrestrial carnivore species. In a striking contrast, RABV is found only in terrestrial hosts and has rarely been isolated from any species of bats in Europe, Africa, Eurasia, and Australasia thus far; however, other lyssaviruses continue to be occasionally found in chiropteran species in these areas [1-3]. 
India is endemic for canine rabies and accounts for almost a third of the 60000 global human deaths due to rabies [4]. Most human deaths in India are attributed to exposure to rabid dogs. No other lyssaviruses except RABV has been reported from India. Systematic surveillance for rabies in humans and animals is not done in India and no attempts for active or passive surveillance for rabies in bats have been made. Bat rabies has not been conclusively proven in India and hence, post-exposure prophylaxis (PEP) for rabies following exposure to bats is currently not recommended in India [5].

Nagaland, a north-eastern state in India bordering Myanmar is known for its unique landscape and rich biodiversity. However, high hunting pressure and a fast-shrinking forest has been a cause of concern in this area [6]. Traditionally, every year thousands of cave-dwelling bats are hunted for consumption as well as to make traditional medicines in some villages in Nagaland. For instance, in Mimi village near the Indo-Myanmar border, every year around mid-October, a bat harvest is organized and within 2 days, about 7000-25000 bats are harvested for consumption from various caves [7]. In other parts of Nagaland also, hunting of bats is quite prevalent, though no other traditional harvests are reported.

A majority of emerging infectious diseases are known to be zoonotic in origin and bats have been implicated as hosts and reservoirs for several of them. It is therefore particularly important to carry out surveillance in regions where there is high biodiversity and at the same time intense human interaction with wildlife through land-use change and hunting, which can facilitate the movement of pathogens to humans.

We visited the bat harvesting festival during two consecutive years - October 2013 and 2014 - and sampled bats in five locations in Nagaland, India to screen bats for any evidence of infection with RABV.

\section{METHODS}

The sampling protocol was approved by the IAEC (Institutional Animal Ethics Committee) at NCBS (National Centre for Biological Sciences), Bangalore.

Sampling of bats was done in areas where traditionally bats are hunted for consumption. A meeting was arranged with the village councils and all the clan members to explain our study. We also conducted multiple outreach programs in villages addressing the ecological importance of bats as well as the potential impact to their health. We obtained verbal permission from the clan leaders to witness the bat harvest/hunting, interview some of the clan members and collect samples for the study. Villagers were requested to submit their hunted bats for sampling on a voluntary basis and were compensated for it.

The five locations in Nagaland where bat sampling was done were Mimi in Kiphire district $\left(25^{\circ} 42^{\prime} 38.01^{\prime \prime}\right.$ N 94 $54^{\prime} 22 \cdot 72^{\prime \prime} \mathrm{E}$ ); Medziphema town, in Dimapur district, located at $25^{\circ} 45^{\prime} 23 \cdot 31^{\prime \prime} \mathrm{N}, 093^{\circ} 52^{\prime} 53 \cdot 27^{\prime \prime} \mathrm{E}$, with an average elevation of about $500 \mathrm{~m}$ above sea level; Salomi village in Kiphire district, located at an

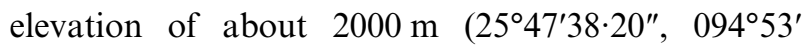
$\left.37.96^{\prime \prime}\right)$; Zubza in Kohima district $\left(25^{\circ} 42^{\prime} 12 \cdot 12^{\prime \prime} \mathrm{N}\right.$ $\left.94^{\circ} 01^{\prime} 31.47^{\prime \prime} \mathrm{E}\right)$; and Reguri in Phek district $\left(25^{\circ} 30^{\prime}\right.$ 24.9948"N 94³8'38.42"E).

In Mimi village, traditionally mass harvesting of bats is done only once a year for 1-2 days. The caves are smoked and the outlets are blocked so that the bats suffocate to death and are collected by the villagers. Due to rugged terrain of the harvesting sites it takes about $1-2 \mathrm{~h}$ for the bats to be brought to the village by the hunters. Hence, blood could not be obtained and only brain tissues from bats in this location could be sampled.

In Salomi and Reguri villages, bamboo sticks or tree twigs are used by villagers to immobilize and kill bats. Bat hunting is carried out throughout the dry season (October to February). In Zubza and Medziphema villages, bats are occasionally hunted by villagers using catapults. Additionally, we opportunistically surveyed and trapped bats using mist nets in these areas, and sacrificed them for adequate sampling. In these locations brain tissues and blood samples could be collected within a few minutes after they were hunted (Figs 1, 2 and 3).

Polystyrene boards wrapped with plastic films were used to pin and dissect the bats. Blood samples were collected by cardiac puncture in $1.5 \mathrm{ml}$ microcentrifuge tubes. A sterile surgical blade was used to cut the skull open to extract the brain into containers with glycerol saline. Serum was separated using a mini centrifuge, aliquoted and stored in an ice-box. All samples were transported to the laboratory in a cold chain. Bats were identified using keys from Bates and Harrison, 1997 [8].

Samples were processed in the BSL-3 (biological safety level 3) facility at the Department of Neurovirology, NIMHANS (National Institute of Mental Health \& Neurosciences). All bat brains $(n=164)$ were subjected to the fluorescent antibody 


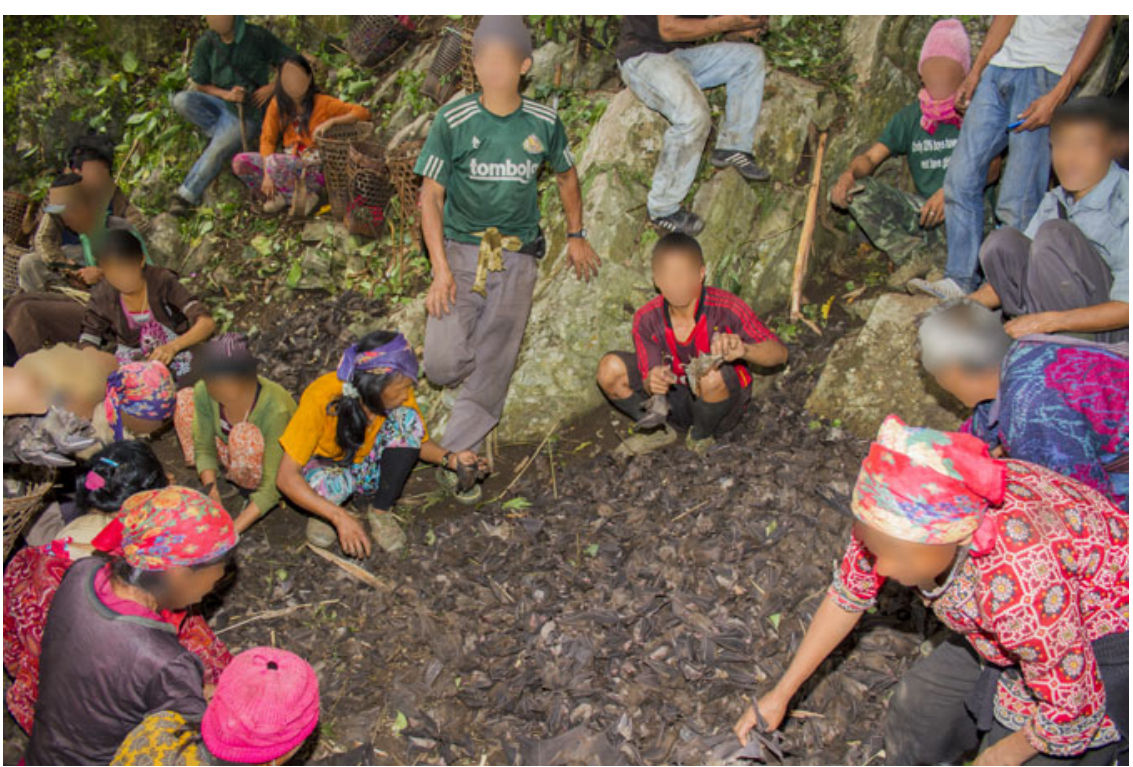

Fig. 1. Villagers sorting the harvested bats at Mimi village, Nagaland.

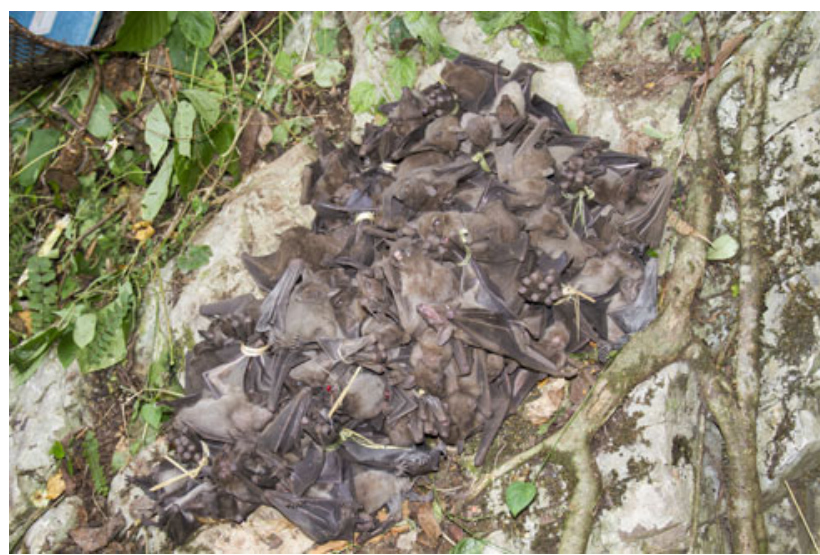

Fig. 2. Harvested bats packed in bundles to be transported home.

test (FAT) for detection of rabies viral antigen [9] using commercial FITC (fluorescein isothiocyanate) conjugated anti-rabies monoclonal antibodies (Light Diagnostics, EMD Millipore Corporation, Temecula, CA) and real-time PCR for detection of rabies viral RNA as described earlier [10, 11].

Sera were collected from 90 bats; only 78 were found to have adequate quantity to be processed for detection of rabies viral neutralizing antibodies (RVNA) using the RFFIT (rapid fluorescent focus inhibition test) according to the World Health Organization (WHO) recommended procedure [12] with some modifications [13]. Briefly, BHK 21 cell line (ATCC CCL 10) and BHK 21 adapted CVS 11 strain of virus were used and the tests were performed in 96 well-tissue culture plates. The antibody titers were expressed in international units $(\mathrm{IU} / \mathrm{ml})$ in comparison to an in-house reference serum calibrated against second International Reference Serum obtained from National Institute of Biological Standards, UK. The lower limit of detection of the assay was $0 \cdot 1 \mathrm{IU} / \mathrm{ml}$.

\section{RESULTS}

Samples of bats belonging to seven species from various locations in Nagaland were obtained; the common species found and sampled included Rousettus leschenaultii (Leschenault's rousette), Hipposideros armiger (Great Himalayan leaf-nosed bat), Eptesicus serotinus (Serotine bat), and Cynopterus sphinx (Greater short-nosed fruit bat) (Tables 1 and 2). 


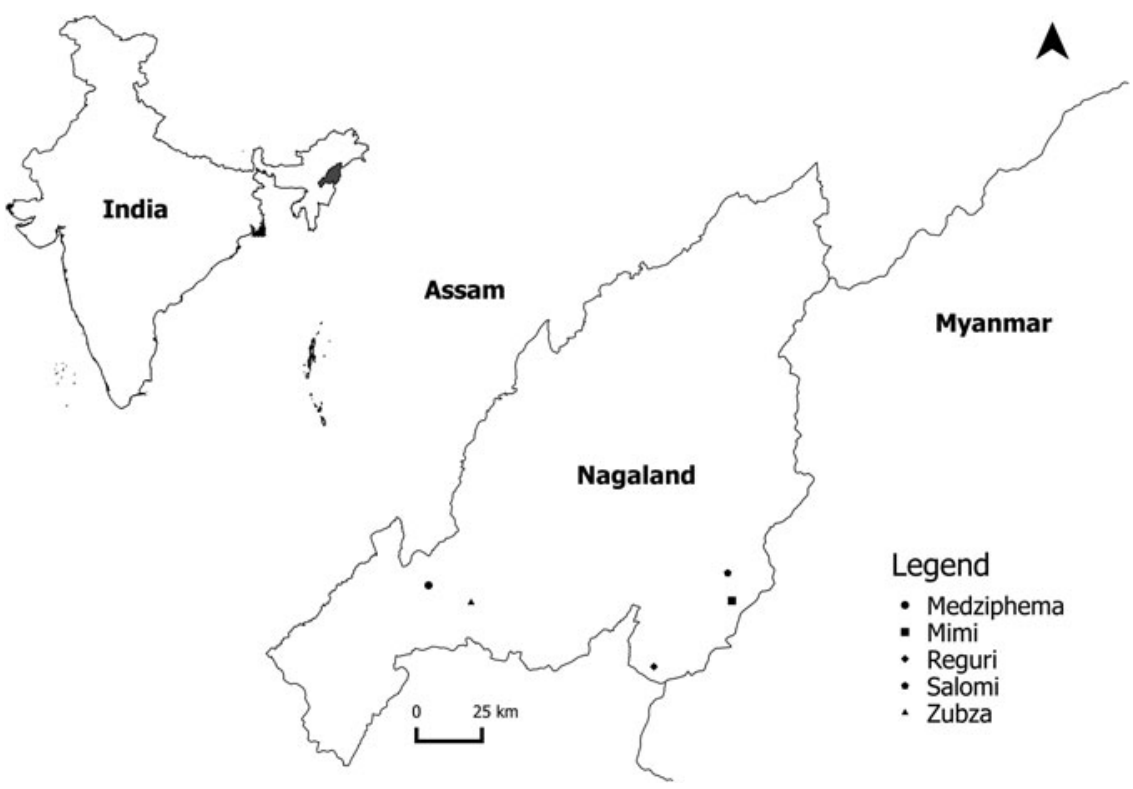

Fig. 3. Locations in Nagaland where bats were sampled for testing.

None of the 164 bat brain tissues tested was positive for rabies nucleoprotein antigen by FAT or viral RNA by real-time PCR (Table 1). RVNA (titers $>0.5 \mathrm{IU} / \mathrm{ml})$ were detected in $4 / 78(5.1 \%)$ samples tested. Only two species of bats, $C$. sphinx and $E$. serotinus were positive for RVNA (Table 2). The RVNA titers detected were $0.9 \mathrm{IU} / \mathrm{ml}$ for one serum sample each from E. serotinus and C. sphinx and 1.8 $\mathrm{IU} / \mathrm{ml}$ in sera obtained from two C. sphinx bats.

\section{DISCUSSION}

A majority of the 14 known species of lyssaviruses, including RABV have been detected in bats, and evolutionary analyses indicate that all lyssaviruses most likely originated in bats $[1,14,15]$. However, there is limited information about RABV and other lyssaviruses in bats in Asia due to lack of systematic surveillance. Several species of bats in the New World are known to harbor variants of RABV, and RABV-infected insectivorous bats are currently responsible for most human cases of rabies in North America. In contrast, bats of the Old World have rarely been reported to carry $\mathrm{RABV}$ either by antigen detection or virus isolation, but highly divergent non-RABV isolates have been reported from few bat species [1,3]. Evidence of active lyssaviral infection has been reported in an Indian flying fox (Pteropus poliocephalus) from northern India [16] and human infections from uncharacterized lyssaviruses acquired through bat bites were reported from India [17] and
Table 1. Bat brains tested from various locations in Nagaland, India for detection of rabies viral antigen and nucleic acid $(n=164)^{*}$

\begin{tabular}{|c|c|c|c|c|}
\hline \multirow[b]{2}{*}{ Bat species } & \multicolumn{3}{|c|}{ Location } & \multirow[b]{2}{*}{ Total } \\
\hline & Salomi & Mimi & Medziphema & \\
\hline Rousettus leschenaulti & & 79 & & 79 \\
\hline Hipposideros armiger & 3 & 24 & & 27 \\
\hline Eonycteris spelaea & & 8 & & 8 \\
\hline Eptesicus serotinus & 19 & & & 19 \\
\hline Pipistrellus affinis & 5 & & & 5 \\
\hline Cynopterus sphinx & & & 12 & 12 \\
\hline Rhinolophus macrotis & 14 & & & 14 \\
\hline Total & 41 & 111 & 12 & 164 \\
\hline
\end{tabular}

* All 164 bat brains tested were negative for rabies viral antigen and nucleic acid.

China [18]. However, these events were not corroborated by any additional reports.

None of the 164 bat brain samples tested in this study was positive for rabies antigen or viral RNA suggesting the absence of an active rabies infection, comparable to other studies in Asia [19-22]. Active bat surveillance for RABV and/or other lyssaviruses in several other countries in Europe or Latin America have also reported a very low prevalence of viral antigen or nucleic acid in healthy bat populations [23-26]. The brain samples were not tested for non-RABV lyssaviral infections in our study. The primers and probe used in this study were reportedly able 
Table 2. Results of Bat sera tested from for rabies virus neutralizing antibodies from various locations (positive) number of sera tested)

\begin{tabular}{llllll}
\hline \hline & \multicolumn{2}{l}{ Location } & & & \\
\cline { 2 - 4 } Bat species & Salomi & Zubza & Medziphema & Reguri & Total \\
\hline $\begin{array}{l}\text { Eptesicus serotinus } \\
\text { Hipposideros armiger }\end{array}$ & $1 / 14$ & $0 / 2$ & & & $1 / 14$ \\
$\begin{array}{l}\text { Pipistrellus affinis } \\
\begin{array}{l}\text { Cynopterus sphinx } \\
\text { Rhinolophus macrotis }\end{array}\end{array}$ & $0 / 4$ & & & $0 / 8$ & $0 / 10$ \\
Total & $0 / 10$ & $1 / 8$ & $2 / 32$ & & $0 / 4$ \\
\hline \hline
\end{tabular}

to detect a few other lyssaviruses, however, they were not found to be optimal for detection of lyssaviruses other than RABV [11].

However, 4/78 (5.1\%) serum samples had evidence of neutralizing antibodies against RABV suggestive of prior exposure to RABV or related lyssaviruses. Only two species of bats, C. sphinx and E. serotinus were found to be seropositive. A few additional sera were positive at low titers, however, only bats with titers above $0.5 \mathrm{IU} / \mathrm{ml}$ were considered seropositive for increased specificity [19, 27], although no accepted standard for naturally occurring infection among bats and other wildlife is available.

Neutralizing antibodies against several lyssaviruses in both insectivorous and frugivorous bats in Asia has been reported from Cambodia, the Philippines, Thailand, Bangladesh, China, and Vietnam. However, neutralizing antibodies against RABV has been reported in bats only from southern China, northern Vietnam, and Cambodia to-date [19-22, 28, 29].

In Cambodia, of the 146 bat sera tested, 30 (20.5\%) were positive for neutralizing antibodies against at least one of the four lyssaviruses tested, with the highest seroprevalence seen in frugivorous species $C$. sphinx and Pteropus lylei. Six $(4 \cdot 1 \%)$ bat sera were positive for neutralizing antibodies against RABV [19]. In southern China, $15(2 \cdot 2 \%)$ of 685 bat sera from three bat species were positive for RABV antibodies, predominantly in frugivorous species $R$. leschenaultii [29]. Ninety $(11.4 \%)$ of 789 bat sera tested in northern Vietnam in a recent study had neutralizing antibodies against RABV, mainly from insectivorous bats [21].

Bats exposed to aerosolized RABV in experimental studies did not demonstrate evidence of disease, but developed demonstrable neutralizing antibodies and survived [30]. Constant vocalization and frequent echolocation in densely packed colonies are potential sources of aerosols from rabies-infected bats which may account for the seropositivity in several bat species across the world, despite absence of active disease. A robust serological response following experimental infection in seronegative bats or survival of seronegative bats despite subsequent challenge with infective doses of virus may indicate the presence of low level of antibodies below the threshold of detection using current tests [1].

Bats are known to harbor several viral pathogens and the recent detection of filoviruses [31], coronaviruses [32], and henipaviruses [33] has kindled further interest in bats as a source of emerging viral infectious diseases. Studies on a bat variant of RABV suggest the enhanced ability of even a small dose of the virus, to be transmitted through a bite and cause lethal disease, due to its ability to replicate in epidermal cells on the body surface and at lower temperatures [34]. The perceived high risk of rabies transmission has led to the recommendation of PEP following any encounter with insectivorous bats in USA.

Despite the constant threat of emerging infectious diseases, there is a lack of systematic human and animal disease surveillance in India. In the north-eastern state of India where this study was conducted, bats are handled with bare hands by the locals during the bat harvesting festival. Furthermore, consumption of these bats may also pose a significant threat to human health. C. sphinx which was detected to be positive for the RABV antibodies was from the suburbs of Dimapur, one of the busiest commercial cities in Northeast India. The high human density in urban areas may provide a greater opportunity of exposure to infected bats and potential disease outbreaks. Additionally, the cave roosting species such as E. serotinus, Rhinolophus macrotis, Pipistrellus affinis, Rousettus leschenaulti, H. armiger, and Eonycteris spelaea which usually have clustered roosting behavior can easily transmit viral infections, both intra- and 
interspecies. Anthropogenic interference such as disrupting bat roosting caves and hunting may influence disease transmission.

Limitations of this study include a small sample size, non-representative sampling and lack of longitudinal sampling. Moreover, cross-reactivity to other unreported/unknown circulating lyssaviruses cannot be ruled out in positive samples. E. serotinus bats which are a very adaptable species and can thrive in a varying range of habitats are considered as the primary reservoir hosts for European bat lyssavirus 1 (EBLV-1) in Europe. EBLV-1 reportedly demonstrates a host restriction to serotine bats and studies indicate that serotine bats may be infected with EBLV-1 throughout Eurasia [3, 35]. The Aravan and Khujand viruses were isolated from insectivorous bats in central Asia and the Irkut virus first identified in eastern Siberia was later isolated from an insectivorous bat in China. These lyssaviruses included in phylogroup-1 along with RABV, can potentially lead to serological cross-reactivity. Moreover, recently another novel Lyssavirus closely related to RABV (Gannoruwa bat lyssavirus) has been isolated from frugivorous bats in neighboring Sri Lanka [36]. None of these non-RABV lyssaviruses identified elsewhere in Asia or Europe have been reported from India so far; however, since public health measures are directed predominantly towards canine-RABV-mediated human disease, genetic typing of viral isolates is rarely performed, and surveillance for lyssaviruses in bats has never been carried out earlier, the possibility of the presence of other closely related non-RABV lyssaviruses in India cannot be ruled out.

Nevertheless the evidence of lyssaviral antibodies in bats from India, a country with a high burden of human rabies, has important implications in disease transmission and control measures. Cross-species transmission from bats to other non-volant mammals can occur and lyssaviruses can establish onward transmission in the newly infected species [1]. Although the risk of transmission of lyssaviral infection from bats to humans is low, the invariably fatal outcome warrants prompt medical care and control measures. Current WHO guidelines recommend PEP following exposure to bats, considered as category III exposure [4]. Considering bat lyssaviruses have been detected on every continent where bats occur, and there is at least one published record of rabies-like illness after bat bite in India [17], it is prudent to revise the current national guidelines [5] to recommend PEP for bat exposures in India.
Inclusion of a larger sample size, well represented by the diverse species in various geographical areas in India, cross-neutralization tests against other lyssaviruses, and surveillance in sick/dying bats to identify active infection is warranted to better define the prevalence of RABV and other lyssaviral infection in bats.

\section{ACKNOWLEDGEMENTS}

The authors gratefully acknowledge Mr. Makhezho from Mimi village for helping coordinate the field work and Mr. Mahesh and Ms Swarupa Rani for technical help. This work was supported by the Rufford Foundation and Ravi Sankaran Inlaks fellowship for field work.

\section{DECLARATION OF INTEREST}

None.

\section{REFERENCES}

1. Banyard AC, et al. Lyssaviruses and bats: emergence and zoonotic threat. Viruses 2014; 6: 2974-2990.

2. Bourhy $\mathbf{H}$, et al. Antigenic and molecular characterization of bat rabies virus in Europe. Journal of Clinical Microbiology 1992; 30: 2419-2426.

3. Banyard AC, et al. Bat rabies. In: Jackson AC, ed. Rabies-Scientific Basis of Disease and its Management, 3rd edn. Oxford: Academic Press, 2013, pp. 215-268.

4. World Health Organization. WHO Expert Consultation on Rabies: Second Report. World Health Organization Technical Report Series 982, Geneva: WHO, 2013.

5. National Centre for Disease Control. National guidelines on rabies prophylaxis. 2015. (http://www.ncdc.gov.in/ writereaddata/mainlinkfile/File557.pdf). Accessed 23 April 2016.

6. Reddy SC, et al. Quantification and monitoring of deforestation in India over eight decades (1930-2013). Biodiversity and Conservation 2015; 1: 1-24.

7. Dovih P. Can traditional bat hunts cause disease outbreaks in India? Economic and Political Weekly 2015; 50: $13-16$.

8. Bates PJJ, Harrison DL. Bats of the Indian Subcontinent. Sevenoaks, Kent, UK: Harrison Zoological Museum; 1997.

9. Dean DJ, Abelseth MK, Atanasiu P. The fluorescent antibody test. In: Meslin FX, Koprowsky H, Kaplan MM, eds. WHO Laboratory Techniques in Rabies. 4th edn. Geneva: WHO, 1996, pp. 88-95.

10. Mani RS, et al. Utility of real-time TaqMan PCR for antemortem and postmortem diagnosis of human rabies. Journal of Medical Virology 2014; 86: 1804 1812.

11. Nadin-Davis SA, Sheen M, Wandeler AI. Development of real-time reverse transcriptase polymerase chain 
reaction methods for human rabies diagnosis. Journal of Medical Virology 2009; 81: 1484-1497.

12. Smith JS, Yager PA, Baer GM. A rapid fluorescent focus inhibition test (RFFIT) for determining rabies virus-neutralizing antibody. In: Meslin FX, Kaplan MM, Koprowski H, eds. Laboratory Techniques in Rabies. 4th edn. Geneva: World Health Organization, 1996, pp. 181-191.

13. Ashwath Narayana DH, et al. Safety and immunogenicity study of a new purified chick embryo cell rabies vaccine Vaxirab-N (Pitman-Moore strain) manufactured in India. Human Vaccines and Immunotherapeutics 2014; 10: 120-125.

14. Badrane H, Tordo N. Host switching in Lyssavirus history from the Chiroptera to the Carnivora orders. Journal of Virology 2001; 75: 8096-8104.

15. Streicker DG, et al. Host phylogeny constrains crossspecies emergence and establishment of rabies virus in bats. Science 2010; 329: 676-679.

16. Pal SR, et al. Rabies virus infection of a flying fox bat, Pteropus poliocephalus in Chandigarh, Northern India. Tropical and Geographical Medicine 1980; 32: 265-267.

17. Veeraraghavan N. A Case of Hydrophobia Following Bat Bite. Pasteur Institute of Southern India, Coonoor: Scientific Report 1954, Madras Diocesan Press, p. 40.

18. Tang X, et al. Pivotal role of dogs in rabies transmission, China. Emerging Infectious Diseases 2005; 11: 19701972.

19. Reynes JM, et al. Serologic evidence of Lyssavirus infection in bats, Cambodia. Emerging Infectious Diseases 2004; 10: 2231-2234.

20. Kuzmin IV, et al. Lyssavirus surveillance in bats, Bangladesh. Emerging Infectious Diseases 2006; 12: 486-488.

21. Nguyen AT, et al. Bat lyssaviruses, northern Vietnam. Emerging Infectious Diseases. 2014; 20: 161-163.

22. Arguin PM, et al. Serologic evidence of Lyssavirus infections among bats, the Philippines. Emerging Infectious Diseases 2002; 8: 258-262.

23. Schatz J, et al. Twenty years of active bat rabies surveillance in Germany: a detailed analysis and future perspectives. Epidemiology and Infection 2014; 142: 1155-1166.

24. Picard-Meyer E, et al. Active surveillance of bat rabies in France: a 5-year study (2004-2009). Veterinary Microbiology. 2011; 151: 390-395.

25. Nokireki T, et al. Bat rabies surveillance in Finland. BMC Veterinary Research 2013; 9: 174.

26. Ellison JA, et al. Bat rabies in Guatemala. PLoS Neglected Tropical Diseases 2014; 31: e3070.

27. Casagrande DK, et al. Rabies surveillance in bats in Northwestern State of São Paulo. Revista da Sociedade Brasileira de Medicina Tropical 2014; 47: 709-715.

28. Lumlertdacha B, et al. Survey for bat lyssaviruses, Thailand. Emerging Infectious Diseases 2005; 11: 232 236.

29. Jiang Y, et al. Seroprevalence of rabies virus antibodies in bats from southern China. Vector Borne and Zoonotic Diseases 2010; 10: 177-181.

30. Davis AD, Rudd RJ, Bowen RA. Effects of aerosolized rabies virus exposure on bats and mice. Journal of Infectious Diseases 2007; 195: 1144-1150.

31. Towner JS, et al. Isolation of genetically diverse Marburg viruses from Egyptian fruit bats. PLoS Pathogens 2009; 5: e1000536.

32. Shirato K, et al. Detection of bat coronaviruses from Miniopterus fuliginosus in Japan. Virus Genes 2012; 44: $40-44$.

33. Halpin $\mathbf{K}$, et al. Pteropid bats are confirmed as the reservoir hosts of henipaviruses: a comprehensive experimental study of virus transmission. American Journal of Tropical Medicine and Hygiene 2011; 85: 946-951.

34. Morimoto $\mathbf{K}$, et al. Characterization of a unique variant of bat rabies virus responsible for newly emerging human cases in North America. Proceedings of the National Academy of Sciences USA 1996; 93: 56535658.

35. Schatz $\mathbf{J}$, et al. Bat rabies surveillance in Europe. Zoonoses Public Health 2013; 60: 22-34.

36. Gunawardena PS, et al. Lyssavirus in Indian Flying Foxes, Sri Lanka. Emerging Infectious Diseases 2016; 22: $1456-1459$. 